\title{
Clinical performance of Non-invasive prenatal testing for sex chromosomal aneuploidies in Northeast China
}

\section{Lu Wang}

Jilin University First Hospital

\section{Rulin Dai}

Jilin University First Hospital

\section{Qingyang Shi}

Jilin University First Hospital

\section{Yuting Jiang}

Jilin University First Hospital

\section{Hongguo Zhang}

Jilin University First Hospital

\section{Ruizhi Liu}

Jilin University First Hospital

Qi Xi ( $\nabla$ cmu_xiqi@163.com )

The First Hospital, Jilin University

\section{Research}

Keywords: Non-invasive prenatal testing (NIPT), Sex chromosomal aneuploidies (SCAs), Positive predictive value (PPV), False positive rate (FPR)

Posted Date: August 3rd, 2020

DOl: https://doi.org/10.21203/rs.3.rs-47409/v2

License: (9) (1) This work is licensed under a Creative Commons Attribution 4.0 International License. Read Full License 


\section{Abstract}

Background: Along with the discovery of cell-free DNA (cfDNA) and the invention of next-generation sequencing (NGS), non-invasive prenatal testing (NIPT) had appeared and been applied for detecting common aneuploidies such as trisomy 21,18 , and 13 , with low false-negative and false-positive rates. Recently, it had also been used for sex chromosome aneuploidies (SCAs). To assess the clinical utility of NIPT for SCAs in Northeast China, we collected NIPT data from BGI 500 sequencing platform in the Center for Reproductive Medicine, Center for Prenatal Diagnosis of the First Hospital of Jilin University, and calculate the positive predictive value (PPV) and false positive rate (FPR).

Results: A cohort of 14936 samples were analyzed by NIPT, and revealed 70 cases with SCAs high-risk, among them, 40 women agreed to undergo amniocentesis, but as many as 30 ones refused further diagnose. Based on verified fetal karyotype, $30.0 \%$ (12/40) were confirmed to be a true positive. Unluckily, the PPV for monosomy X performed $0 \%$. Besides, positive 47,XXX were $46.67 \%$ (7/15), 40.00\% (2/5) were positive for 47, $X Y Y$, and $42.86 \%$ (3/7) were positive for $47, \mathrm{XXY}$.

Conclusions: In conclusion, our present results confirmed that NIPT sequenced by BGI 500 demonstrated lowest PPVs for $45, X$, but the more accurate prediction for other SCAs, it is still a potential method for SCAs screening. Henceforth, we should focus on how to improve the test utility and provide better services for pregnant women in need.

\section{Background}

With high sensitivity and specificity, non-invasive prenatal testing (NIPT) is an unparalleled screening method for fetal aneuploidies [1]. As we know, it has now been widely used in the clinical screening for trisomy diseases, such as Patau, Edwards, and Down syndrome [2,3], and the analysis of cell-free DNA (cfDNA) in maternal blood showed high accuracy in trisomy 21, 18, and 13 [4]. However, false-positive, false-negative, and failure-reportable cases can take place, and which may have some biological reasons.

Besides these goal aneuploidies, the aneuploidies occurred in the $X$ and $Y$ chromosomes should also be taken seriously. In 2012, NIPT of sex chromosome aneuploidy (SCAs) was introduced in the United States. At first, there was little information on the effects of these trials; however, its development was rapid $[5,6]$. The influence of SCAs containing Turner $(45, X)$, Klinefelter $(47, X X Y)$, Jacob syndromes $(47, X Y Y)$ and Triple $X$ syndrome $(47, X X X)$ on new births is about $0.3 \%[7,8,9]$. Turner, Klinefelter and some cases of $47, X X X$ could lead to delayed or abnormal sexual growth, and infertility, especially Turner and Triple $X$ syndrome might show mental retardation [10]. To grasp the correct diagnostic information of neonatal SCAs is very important for early treatment. For many cases of SCAs, initial screening results in a better prognosis [11].

Nowadays, most laboratories provided the results of the SCAs as part of the report. However, recent researches had shown that the NIPT is less accurate in SCAs detection than autosomal aneuploidy [12,13]. But, a few of false-positive cases and low positive predict value still existed.

Our institution set up BGISEQ-500 sequencing platform in 2018, which was powered by Combinatorial ProbeAnchor Synthesis (CPAS) and modified DNA Nanoballs (DNBs) methods. In previous studies, BGISEQ-500 is a recently launched high-through put sequencing protocol; NIPT based on CPAS seemed to be a suitable method for 
routine clinical screening for SCAs [14]. Therefore, in our study, we try to investigate the accuracy of NIPT in SCAs screening among pregnancies admitted to our lab from Oct 2018 to May 2020, to evaluate our clinical performance of CPAS in detecting SCAs.

\section{Materials And Methods}

\section{Subjects}

A number of 14936 samples from pregnant women who visited at our institution, between Oct 2018 and May 2020 accepted NIPT. After the report, 40 patients of 70 SCAs high-risked cases were undergone amniocentesis for further prenatal diagnosis.

Written informed consent had been signed after the nature of the procedures had been fully explained before the study. The Medical Ethics Committee of First Hospital of Jilin University approved our research (No.2018-441).

\section{NIPT}

Maternal peripheral blood samples were collected in Cell-Free DNA Storage Tube (CWBIO, Taizhou, China), and centrifuged within 96 hours. The plasma was separated and stored at $-80^{\circ} \mathrm{C}$ until cfDNA was extracted. cfDNA was obtained from $200 \mu$ l plasma with Nucleic Acid Extraction Kit by using BGISP-300 (BGI, Shenzhen, China). And library DNA was constructed by Fetal Chromosome Aneuploidy (T21, T18, T13) Detection Kit (Combined probe-anchored polymerization sequencing method). The products were quantified by Qubitß 3.0 Fluorometer (Life Technologies, Invitrogen USA) using Qubit ${ }^{T M}$ dsDNA HS Assay Kits and the concentration $\geq 8 n g / \mu l$ was qualified standards. Each sample's volume was calculated according to the concentration, to make sure the same mass is mixed into a pool. After pooling, the qualified DNBs (appropriate range from 8 to $40 \mathrm{ng} / \mu \mathrm{l}$ ) were produced and loaded onto chips, then sequenced on BGISEQ-500 (BGI, Shenzhen, China).

\section{Karyotype and Chromosome microarray analyses of amniotic fluid cells}

NIPT positive patients were recommended undergoing amniocentesis to examine fetal chromosomes.

Amniocentesis was performed at 16-23 weeks of gestation to extract amniotic fluid cells, $25 \mathrm{ml}$ amniotic fluid cells were cultured in amniotic membrane medium (Irvine Scientific, Santa Ana, CA, USA), then treated with colcemid. Gbanding was performed on metaphase chromosomes by standard method. Each individual had at least 30 metaphase cells counted, and at least 5 cells analyzed. Chromosomal abnormalities were described on the basis of the criteria, which were established by International System for Human Cytogenetic Nomenclature (ISCN 2013). Simultaneously, $10 \mathrm{ml}$ uncultured amino fluid cells were used to detect by Chromosome microarray analyses based on Affymetrix CytoScan 750K array chips (Affymetrix, Santa Clara, CA). Copy number variations (CNVs) and heterozygosis loss (LOH) in the human genome were revealed by $\geq 50$ probe markers and $\geq 200 \mathrm{~Kb}$ resolution, the probe located on 22 pairs of autosomes and sex chromosomes. The results of CNVs and LOH were analyzed by comparing with the public databases or published works of literature.

\section{Statistics}

Online software (http://vassarstats.net/clin1.html) was used to calculate the FPR and PPV, the amniocentesis results regarded as the gold standard, based on the standard normal distribution, the $95 \%$ confidence interval (CI) was calculated by the cloper-Pearson method. 


\section{Results}

NIPT was used to evaluate our fetal DNA in detecting SCAs performance. As a result, the analysis of 14936 pregnancies revealed 70 cases with SCAs high-risk results; the total frequency for the SCAs-positive was $0.50 \%$ (70/14936). Among the 70 abnormal cases, NIPT detected 24cases with Monosomy X (45,X), 27 cases with Trisomy X (47,XXX), 8 cases with $47, X Y Y$ and 11 cases with $47, X X Y$. To verify the accuracy of NIPT positive results, amniocentesis was recommended for verification of abnormal samples. However, of the 70 SCAs-positive cases, 40 women agreed to have a fetal karyotype, but the other 30 refused further examinations for some reasons. The next confirmation according to fetal karyotyping and CMA was available for 40 positive cases (13 for $45, X ; 15$ for $47, X X X ; 5$ for $47, X Y Y$; and 7 for $47, X X Y$; ) and the other 30 patients ( 11 for $45, X ; 12$ for $47, X X X ; 3$ for $47, X Y Y$; and 4 for $47, X X Y$ ) were unconfirmed because the women denied amniocentesis. Compared to the gold standard, none true positive results were found in 13 cases of monosomy $X$; of the 15 patients with trisomy $X, 7$ were true positive, with PPV of $46.67 \%(7 / 15)$. Of the 5 cases of the $47, X Y Y, 2$ cases were true positive, so the PPV of $47, \mathrm{XYY}$ by NIPT was $40.00 \%$ (2/5). At last, NIPT revealed 47,XXY with a PPV of $42.86 \%$ (3/7), 3 cases of NIPT results were coincident with the diagnose of amniocentesis (Table 1).

At the same time, the comparison of the whole results between NIPT and amniocentesis for fetal SCAs was showed in Table 2. We had known that NIPT screening for Monosomy $X$ in our study showed the highest FPR of all SCAs types, exaggeration to $100 \%$. However, case 1 showed a fetal karyotype of sex chromosome abnormalities (46,XX?) after conventional G-banding, and CMA result explained the abnormality of X chromosome was a $0.356 \mathrm{Mb}$ deletion of Xq27, a $0.237 \mathrm{Mb}$ duplication of Xp22 and $0.338 \mathrm{Mb}$ duplication of Xq13. Also, case 2 , case 7 and case 13 were all detected microduplication or microdeletion of $X$ chromosome or autosomes. Case 14-28 in table 2 displayed the comparison of Trisomy X, NIPT, fetal karyotype by G-banding and CMA showed consistent trends in 7 fetuses. About cases with 47, XYY high-risk, in case 29, amniocentesis result not only showed the same karyotype with NIPT but also detected chromosome polymorphism and a microduplication of chromosome 2q. Among 47,XXY high-risked cases 34-40, the false-positive case 39 and case 40 revealed CNVs of autosomes nevertheless. Otherwise, 2 healthy fetuses proved by CMA showed chromosome polymorphism karyotypes of $46, \mathrm{XN}, 1 \mathrm{qh}+$ and $46, \mathrm{XN}, 21 \mathrm{pss}$ in case 8 and case23.

\section{Discussion}

The sensitivities for T21, T18, and T13 had reached greater than 99\% [15]. However, as far as sex chromosomes were concerned, aneuploidy detection remained problematic, as some previous studies had shown $[5,6,16,17]$. This time, we focused on SCAs detected by BGISEQ-500 sequencing platform. Among the 14936 cases, 70 cases showed sex chromosome abnormality by NIPT. Karyotype analysis of 40 SCAs cases; the consistency rate of amniotic fluid was about $30.00 \%$. The detection rate was different from previous tests, so the positive predictive value was used as a more accurate method. And thus, concrete SCAs' PPV is all be counted (45,X=0\%, $47, X X X=46.67 \%, 47 X Y Y=40 \%, 47, X X Y=42.86 \%)$, from the results, 45,X detection seemed to be none accurate. Similarly, in 2018, Li et al. reported a series of SCAs' PPV by the same methods with us, ranged from 47,XXX (90\%, $\left.\mathrm{Cl}_{95}, 55.95-98.46 \%\right), 47, \mathrm{XXY}\left(75 \%, \mathrm{Cl}_{95}, 49.25-90.27 \%\right), 47, \mathrm{XYY}\left(50 \%, \mathrm{Cl}_{95}, 12.37-87.63 \%\right)$ to $45, \mathrm{X}\left(36.84 \%, \mathrm{Cl}_{95}\right.$ ,25.00-50.52\%). The PPV of 45,X was the lowest, too[18]. Wang et al. also showed their PPV of SCAs sequenced by BGISEQ-500, the PPV in total SCAs was 19/33(57.6\%), the specific PPV of 45,X was 3/14(21.4\%), 47,XXX $3 / 4(75.0 \%), 47, X X Y 10 / 11(90.9 \%), 47, X Y Y 3 / 4(75.0 \%)$ [19]. All data indicated that the FPR of 45,X was still the highest based on BGISEQ-500, and the accuracy needed to be improved. 
Several reasons might explain the poor PPV of monosomy X: at first, as we know, there were 1098 genes located on the $X$ chromosome, but $Y$ chromosome contained only 78 genes, among them, there were also homologous 58 genes on both $X$ chromosomes and $Y$ chromosomes, and 29 genes were at the ends of sex chromosomes. Secondly, the sequencing read length was only 35 bases, which might easily lead to the misplacing of $X$ and $Y$ chromosome. The non-random inactivation of $X$ chromosomes in placental tissues may be the third reason, in $X X$ female trophoblast cells, $X$ chromosomes from father tended to inactivate more easily $[20,21,22]$.

Although, the current NIPT could detect fetal 21, 18 and 13 trisomies, with high sensitivity and specificity [23,24]. But, a small number of tests could give false-positive results or a complete failure for several reasons, including maternal chromosomal mosaicism [25], confined placental mosaics [26], maternal malignancy [2,27,28,29], cotwin demise [30], and so on. In the same way, these causes could also lead to false positive results for SCAs. Generally speaking, our results of all types SCAs performed on the lower side than the above mentioned, because of fewer subjects $(57.14 \%, 40 / 70)$ would to accept invasive surgery, who afraid of miscarriage or intrauterine infection, its small size limited our study, in the future, we'll keep watching and updating the results.

Otherwise, it is worth mentioning that among our revealed false-positive SCAs cases, 2 cases were found CNVs of the $\mathrm{X}$ chromosome, and there were 3 cases found with autosomal CNVs detected by CMA, including microdeletion and microduplication. It is worth considering whether other fetal CNVs will cause different NIPT risk values to increase, and it is necessary to carry out CMA and karyotype analysis simultaneously, if possible.

\section{Conclusion}

NIPT powered by BGI 500 sequencing platform yielded the lower PPVs for SCAs, especially for 45,X. Forecast of other SCAs was more accurate. However, this technique needed further research. Invasive prenatal karyotype analysis should be recommended for pregnant women with fetal SCAs, and CMA ran in parallel if possible. Clinicians should fully inform patients of the limitations of NIPT and make it clear that it is only a prenatal screening technology. Fetal health should be evaluated in combination with other measures.

\section{Declarations}

\section{Acknowledgements}

We thank all the subjects participating in this work for their informed consents, and thanks to the leader and founder of NIPT field, too.

\section{Author's contribution}

Lu Wang, Rulin Dai and Yuting Jiang performed the clinical data collection and analysis. Qingyang Shi performed genetic counseling and amniocentesis. Karyotype analysis was performed by Hongguo Zhang. The first draft was written by Lu Wang, and Ruizhi Liu was responsible for revision, at last, Qi Xi submitted this article.

\section{Funding}

This research is supported by the Finance Department Health Special Project of Jilin Province, China. (JLSCZD2019-022) 
All data generated or analyzed in this study are presented in the article.

\section{Competing interest}

The authors state that they have no conflict of interest.

\section{Ethics approval and consent to participate}

Written informed consent was obtained from all patients participating, and our study was authorized by the Ethics Committee of the First Hospital of Jilin University. (No.2018-441)

\section{Consent for publication}

All patients agreed to authorize our institution to use their samples, phenotypic information and testing data.

\section{Abbreviations}

NIPT: non-invasive prenatal testing; cfDNA: cell-free DNA; NGS: next-generation sequencing; SCAs: sex chromosome aneuploidies; PPV: positive predictive value; FPR: false positive rate. CPAS: Combinatorial ProbeAnchor Synthesis; DNBs: DNA Nanoballs; CMA: Chromosome microarray analyses; CNVs: Copy number variations; LOH: heterozygosis loss; ISCN: International System for Human Cytogenetic Nomenclature.

\section{References}

1. Hartwig TS, Ambye L, Sørensen S, Jørgensen FS. Discordant non-invasive prenatal testing (NIPT) - a systematic review. Prenat Diagn. 2017;37(6):527-539.

2. Lo YM. Non-invasive prenatal diagnosis by massively parallel sequencing of maternal plasma DNA. Open Biol. 2012;2(6):120086.

3. Fan HC, Blumenfeld YJ, Chitkara U, Hudgins L, Quake SR. Noninvasive diagnosis of fetal aneuploidy by shotgun sequencing DNA from maternal blood. Proc Natl Acad Sci U S A. 2008;105(42):16266-16271.

4. Samura O, Okamoto A. Causes of aberrant non-invasive prenatal testing for aneuploidy: A systematic review. Taiwan J Obstet Gynecol. 2020;59(1):16-20.

5. Ramdaney A, Hoskovec J, Harkenrider J, Soto E, Murphy L. Clinical experience with sex chromosome aneuploidies detected by noninvasive prenatal testing (NIPT): Accuracy and patient decision-making. Prenat Diagn. 2018;38(11):841-848.

6. Reiss RE, Discenza M, Foster J, Dobson L, Wilkins-Haug L. Sex chromosome aneuploidy detection by noninvasive prenatal testing: helpful or hazardous?. Prenat Diagn. 2017;37(5):515-520.

7. Nielsen J, Wohlert M. Chromosome abnormalities found among 34,910 newborn children: results from a 13year incidence study in Arhus, Denmark. Hum Genet. 1991;87(1):81-83.

8. Jacobs PA, Browne C, Gregson N, Joyce C, White H. Estimates of the frequency of chromosome abnormalities detectable in unselected newborns using moderate levels of banding. J Med Genet. 1992;29(2):103-108.

9. Morris JK, Alberman E, Scott C, Jacobs P. Is the prevalence of Klinefelter syndrome increasing? Eur J Hum 2008;16(2):163-70. 
10. Pieters JJ, Kooper AJ, van Kessel AG, Braat DD, Smits AP. Incidental prenatal diagnosis of sex chromosome aneuploidies: health, behavior, and fertility. ISRN Obstet Gynecol. 2011;2011:807106.

11. Abramsky L, Hall S, Levitan J, Marteau TM. What parents are told after prenatal diagnosis of a sex chromosome abnormality: interview and questionnaire study. BMJ. 2001;322(7284):463-466.

12. Chiu RW, Akolekar R, Zheng YW, Leung TY, Sun H, Chan KC, et al. Noninvasive prenatal assessment of trisomy 21 by multiplexed maternal plasma DNA sequencing: large scale validity study. 2011;342:c7401..

13. Pan X, Zhang C, Li X, et al. Non-invasive fetal sex determination by maternal plasma sequencing and application in X-linked disorder counseling. J Matern Fetal Neonatal Med. 2014;27(18):1829-1833.

14. Fehlmann T, Reinheimer S, Geng C, et al. cPAS-based sequencing on the BGISEQ-500 to explore small noncoding RNAs. Clin Epigenetics. 2016;8:123.

15. Stokowski R, Wang E, White K, et al. Clinical performance of non-invasive prenatal testing (NIPT) using targeted cell-free DNA analysis in maternal plasma with microarrays or next generation sequencing (NGS) is consistent across multiple controlled clinical studies. Prenat Diagn. 2015;35(12):1243-1246.

16. Wang $Y$, Chen $Y$, Tian F, et al. Maternal mosaicism is a significant contributor to discordant sex chromosomal aneuploidies associated with noninvasive prenatal testing. Clin Chem. 2014;60(1):251-259.

17. Suo F, Wang C, Liu T, et al. Non-invasive prenatal testing in detecting sex chromosome aneuploidy: A largescale study in Xuzhou area of China. Clin Chim Acta. 2018;481:139-141. doi:10.1016/j.cca.2018.03.007

18. Li H, Lei Y, Zhu H, et al. The application of NIPT using combinatorial probe-anchor synthesis to identify sex chromosomal aneuploidies (SCAs) in a cohort of 570 pregnancies. Mol Cytogenet. 2018;11:59.

19. Wang Y, Li S, Wang W, et al. Cell-free DNA screening for sex chromosome aneuploidies by non-invasive prenatal testing in maternal plasma. Mol Cytogenet. 2020;13:10.

20. Eggan K, Akutsu H, Hochedlinger K, Rideout W 3rd, Yanagimachi R, Jaenisch R. X-Chromosome inactivation in cloned mouse embryos. 2000; 290(5496):1578-1581.

21. Clerc P, Avner P. Genetics. Reprogramming X inactivation. Science. 2000; 290(5496):1518-1519.

22. Disteche CM, Berletch JB. X-chromosome inactivation and escape. J Genet. 2015;94(4):591-599.

23. Benn P, Cuckle H, Pergament E. Non-invasive prenatal testing for aneuploidy: Current status and future prospects. Ultrasound Obstet Gynecol. 2013;42:15-33.

24. Chen EZ, Chiu RWK, Sun H, et al. Noninvasive prenatal diagnosis of fetal trisomy 18 and trisomy 13 by maternal plasma dna sequencing. PLOS ONE. 2011;6:1-7.

25. Bianchi DW, Parsa S, Bhatt S, et al. Fetal sex chromosome testing by maternal plasma DNA sequencing: clinical laboratory experience and biology. Obstet Gynecol. 2015;125:375-382.

26. Lau TK, Jiang FM, Stevenson RJ, et al. Secondary findings from non-invasive prenatal testing for common fetal aneuploidies by whole genome sequencing as a clinical service. Prenat Diagn. 2013;33:602-608.

27. Bianchi DW, Chudova D, Sehnert AJ, et al. Noninvasive prenatal testing and incidental detection of occult maternal malignancies. JAMA. 2015;314:162-169.

28. Amant F, Verheecke M, Wlodarska I, et al. Presymptomatic identification of cancers in pregnant women during noninvasive prenatal testing. JAMA Oncol. 2015;1:814-819.

29. Benn P, Plon SE, Bianchi DW. Current Controversies in Prenatal Diagnosis 2: NIPT results suggesting maternal cancer should always be disclosed. Prenat Diagn. 2019;39(5):339-343. 
30. Curnow KJ, Wilkins-Haug L, Ryan A, et al. Detection of triploid, molar, and vanishing twin pregnancies by a single-nucleotide polymorphism-based noninvasive prenatal test. Am J Obstet Gynecol. 2015;212(1):79.e179.e799.

\section{Tables}

Table 1 Efficiency of NIPT in SCAs screening.

\begin{tabular}{|c|c|c|c|c|c|c|c|}
\hline $\begin{array}{l}\text { SCAs } \\
\text { types }\end{array}$ & $\begin{array}{c}\text { NIPT } \\
\text { positive }\end{array}$ & $\begin{array}{c}\text { Karyotype } \\
\text { validated }\end{array}$ & $\begin{array}{c}\text { True } \\
\text { positive }\end{array}$ & $\begin{array}{c}\text { False } \\
\text { positive }\end{array}$ & $\begin{array}{c}\text { Positive } \\
\text { predictive } \\
\text { value }(\mathrm{PPV})\left(\mathrm{CI}_{95}\right)\end{array}$ & $\begin{array}{l}\text { False positive } \\
\text { rate } \\
(\mathrm{FPR})\left(\mathrm{CI}_{95}\right)\end{array}$ & Unknown \\
\hline $45, X$ & 24 & 13 & 0 & 13 & $\begin{array}{c}0.00 \% \\
(0.00 \%-28.34 \%)\end{array}$ & $\begin{array}{c}100 \% \\
(71.66 \%-100 \%)\end{array}$ & 11 \\
\hline $47, \mathrm{XXX}$ & 27 & 15 & 7 & 8 & $\begin{array}{c}46.67 \% \\
(22.28 \%-72.58 \%)\end{array}$ & $\begin{array}{c}53.33 \% \\
(27.42 \%-77.72 \%)\end{array}$ & 8 \\
\hline $47, \mathrm{XYY}$ & 8 & 5 & 2 & 3 & $\begin{array}{c}40.00 \% \\
(7.26 \%-82.96 \%)\end{array}$ & $\begin{array}{c}60 \% \\
(17.04 \%-92.74 \%)\end{array}$ & 3 \\
\hline $47, \mathrm{XXY}$ & 11 & 7 & 3 & 4 & $\begin{array}{c}42.86 \% \\
(11.81 \%-79.76 \%)\end{array}$ & $\begin{array}{c}57.14 \% \\
(20.24 \%-88.19 \%)\end{array}$ & 4 \\
\hline Total & 70 & 40 & 12 & 28 & $\begin{array}{c}30 \% \\
(17.09 \%-46.71 \%)\end{array}$ & $\begin{array}{c}70 \% \\
(53.29 \%-82.91 \%)\end{array}$ & 30 \\
\hline
\end{tabular}

Table 2 The result comparison among NIPT, conventional fetal karyotype by G-banding and CMA. 


\begin{tabular}{|c|c|c|c|}
\hline Case & $\begin{array}{l}\text { NIPT } \\
\text { result }\end{array}$ & Fetal karyotype & CMA result \\
\hline \multirow[t]{2}{*}{1} & $45, \mathrm{X}$ & $46, \mathrm{XX} ?$ & delXq27(0.356 Mb); dupXp22(0.237 \\
\hline & & & 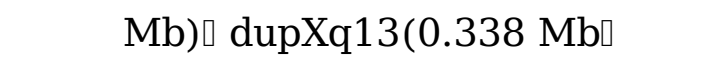 \\
\hline 2 & $45, \mathrm{X}$ & Normal & delXp22.33(0.694 Mb) \\
\hline 3 & $45, \mathrm{X}$ & Normal & Normal \\
\hline 4 & $45, \mathrm{X}$ & Normal & Normal \\
\hline 5 & $45, X$ & Normal & Normal \\
\hline 6 & $45, \mathrm{X}$ & Normal & Normal \\
\hline 7 & $45, \mathrm{X}$ & Normal & dup20p13(0.413Mb) \\
\hline 8 & $45, \mathrm{X}$ & $46, \mathrm{XN}, 1 \mathrm{qh}+$ & Normal \\
\hline 9 & $45, \mathrm{X}$ & Normal & Normal \\
\hline 10 & $45, \mathrm{X}$ & Normal & Normal \\
\hline 11 & $45, X$ & Normal & Normal \\
\hline 12 & $45, X$ & Normal & Normal \\
\hline 13 & $45, \mathrm{X}$ & Normal & dup8q11.23(0.24Mb) \\
\hline 14 & $47, \mathrm{XXX}$ & Normal & Normal \\
\hline 15 & $47, \mathrm{XXX}$ & $47, \mathrm{XXX}$ & $47, \mathrm{XXX}$ \\
\hline 16 & $47, \mathrm{XXX}$ & Normal & Normal \\
\hline 17 & $47, \mathrm{XXX}$ & Normal & Normal \\
\hline 18 & $47, \mathrm{XXX}$ & Normal & Normal \\
\hline 19 & $47, \mathrm{XXX}$ & $47, \mathrm{XXX}$ & $47, \mathrm{XXX}$ \\
\hline 20 & $47, \mathrm{XXX}$ & Normal & Normal \\
\hline 21 & $47, \mathrm{XXX}$ & $47, \mathrm{XXX}$ & $47, \mathrm{XXX}$ \\
\hline 22 & $47, \mathrm{XXX}$ & $47, \mathrm{XXX}$ & $47, \mathrm{XXX}$ \\
\hline 23 & $47, \mathrm{XXX}$ & $46, \mathrm{XN}, 21 \mathrm{pss}$ & Normal \\
\hline 24 & $47, \mathrm{XXX}$ & $47, \mathrm{XXX}$ & $47, \mathrm{XXX}$ \\
\hline 25 & $47, \mathrm{XXX}$ & Normal & Normal \\
\hline 26 & $47, \mathrm{XXX}$ & Normal & Normal \\
\hline 27 & $47, \mathrm{XXX}$ & $47, \mathrm{XXX}$ & $47, \mathrm{XXX}$ \\
\hline 28 & $47, \mathrm{XXX}$ & $47, \mathrm{XXX}$ & $47, \mathrm{XXX}$ \\
\hline 29 & $47, \mathrm{XYY}$ & $47, X Y Y, \operatorname{inv}(9)$ & 47,XYY,dup2q13(0.48Mb) \\
\hline
\end{tabular}


(p11q13)

$\begin{array}{llcc}30 & 47, \mathrm{XYY} & \text { Normal } & \text { Normal } \\ 31 & 47, \mathrm{XYY} & \text { Normal } & \text { Normal } \\ 32 & 47, \mathrm{XYY} & \text { Normal } & 47, \mathrm{XYY} \\ 33 & 47, \mathrm{XYY} & 47, \mathrm{XYY} & \text { Normal } \\ 34 & 47, \mathrm{XXY} & \text { Normal } & 47, \mathrm{XXY} \\ 35 & 47, \mathrm{XXY} & 47, \mathrm{XXY} & 47, \mathrm{XXY} \\ 36 & 47, \mathrm{XXY} & 47, \mathrm{XXY} & \text { Normal } \\ 37 & 47, \mathrm{XXY} & \text { Normal } & 47, \mathrm{XXY} \\ 38 & 47, \mathrm{XXY} & 47, \mathrm{XXY} & \text { dup18p11.31(0.471Mb) } \\ 39 & 47, \mathrm{XXY} & \text { Normal } & \text { del6q26(0.348Mb) } \\ 40 & 47, \mathrm{XXY} & \text { Normal } & \end{array}$

